urethra. The upright portion, on being caught between the two forefingers, separated from the main portion, and was extracted. Litho-1 tomy forceps were then introduced through the wound, and the main portion grasped, but crumbled between the blades on attempting to extract it. Further examination by the finger showed that the lower portion was held in position between the distended rectum and the symphisis pubis, so that it could not be extracted. The rectal bag was, therefore, partially emptied, when the main portion was loosened, caught and extracted. The mortar-like débris that remained was got rid of by repeated injections of boracic solution from a large syringe through the wound. It is wonderful the tenacity with which debris of this kind, embedded in clot, adheres to the walls of the bladder, and great care is necessary to remove every particle of it. Finally, a soft. catheter was passed through the urethra, and a stream of lotion injected through it and out through the wound. The catheter was tied in, and a thick piece of drainage-tube placed in the wound. The npper part of the abdominal wound was brought together by a deep suture, and the whole dressed loosely by lint steeped in corrosive sublimate solution. The débris weighed about an ounce. The shape of the stone and its peculiar position had deceived me as to its size. The arine flowed by the catheter and wound till the second day after the operation, when the catheter was removed, after which it passed altogether by the wound. The abdominal suture soon gave way, and for a few days the margins of the wound were unhealthy-covered by phosphates and superficial slough. Fodoform dressing was used, and the wound soon presented a healthy appearance. The patient also suffered much from diarrhoea. On July 9 th he began to pass urine by the urethra; on the 21st it passed wholly in this way. On August 7 th the wound had completely healed, and the patient was discharged in excellent health.

Though I have had Petersen's rectal bags by me for some three years, this is the first occasion on which I have had to use one, preferring to remove by litholapaxy caleuli as large as 3 ounces in weight. From the fact that I have had recourse to suprapubic cystotomy only once amongst these 100 cases of stone, it may be gathered that I have little sympathy with that recent movement which would relegate to this operation cases of small stone in the bladder. The recent improvements in the method of performing suprapubic cystotomy have, un. doubtedly, rendered it more simple and probably more safe; but 1 look on the operation as a necessary evil, to be had recourse to only in cases of very large stone, or those presenting unusual difficulties, in which neither litholapaxy nor lateral lithotomy can be employed. Amongst recent literature on the subject of stone, I know of none in which more sound views are enunciated regarding the choice of an operation for stone in the bladder than in Mr. Cadge's Lectures, delivered at the Royal College of Surgeons of England, June, 1886.

\section{HYDROCELE IN THE FEMALE.}

BY S. OSBORN, F.R.C.S.

Assistant Surgeon to the Hospital for Women, Soho Square.

HXDROCELe in the female may occur in one of two situations-hydrocele of the femoral canal, or hydrocele of the canal of Nuck. That of the femoral canal is a process of peritoneum projected from the general peritoneal cavity, and not a hydrocele of a femoral sac, where the communication with the peritoneum is shut off by either intestine or omentum becaming adherent to the mouth of the sac. That of the canal of Nuck is a process of peritoneum which accompanies. the round ligament downwards, and extends from the groin to the labium, and which may remain more or less pervious in adult life, and even in some cases has an infinitesimally small opening commu. nicating with the peritoneal cavity. Hydrocele of the femoral canal is more rare than that of the canal of Nuck, and both the cases reported by Mr. Anderson, of Nottingham, and by Dr. Hirst, are of the fatter variety, as also are the two cases read before the New York Clinical Society by Dr. Wright, and reported in the Nevo York Medical Journal.

Several of these cases have at different times come under my notice in the out-patient room of the Soho Hospital. One case of hydrocele of the femoral canal was reported in the Lancet of April 5th 1879 ; and two cases-one of hydrocele of the femoral canal and one of hydrocele of the canal of Nuck-were reported in the Lancet of March $7 \mathrm{thi}, 1885$. "The accompanying woodcut, representing the last of these cases; is one which I had taken for the new edition of my work on this subject which I am now preparing.

Since reporting these last. two cases I have had some six or eight others, and therefore I have not looked upon them as such great rarities as I at one time thought them to be. They are probably not so frequently met with at a general hospital as at a special one, anil undoubtedly are often overlooked or treated as ruptures.

These cases are most liable to be mistaken for an irreducible hernia, and, when inflamed, for strangulated hernia. The application of a truss has been in such cases a means towards cure; but, if the fluid had been first evacuated by tapping, the pad or truss would have fitted more accurately, and the cure been more rapid and more certain.

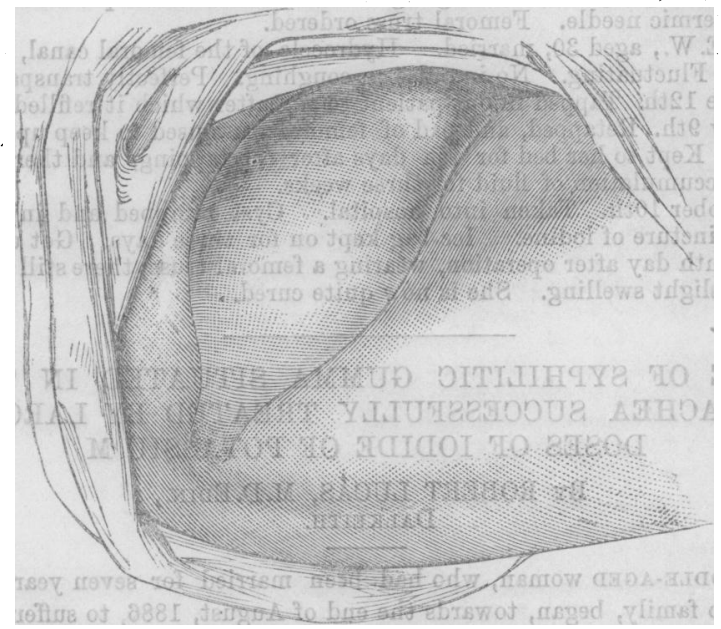

The diagnosis of these cases is not easy ; the difficulty of examining them by transmitted. light is great. An ordinary stethoscope is the best instrument to use for the purpose; but with this care must be taken, as it is very easy to direct the vision against the inner side of the tube, and not directly to its opposite end. An absence of impulse on coughing, and sometimes fluctuation; are other diagnostic signs ; but that of transparency by transmitted light is the only trustworthy one. In doubtful cases the diagnosis can easily be settled by the hypodermic needle. The fluid contained in both varieties is pale yellow or straw-coloured serum.

Tapping, when adopted, should be done at the patient's home, so that subsequent rest in the horizontal position may be maintained. The walls of the cyst should be thoroughly manipulated-that is, rubbed one upon the other; and pressure kept up by means of a truss, or by pad and bandage. This will in some cases effect a cure; but in others I have been compelled to resort to injection of iodine, which is usually successful.

I have hastily selected the following cases from amongst my ontpatient letters :

1. S. B., aged 34, married. Hydrocele of canal of Nuck, right side; noticed only four months; transparent by transmitted light. The patient stated that it was larger some days than others, and sometimes disappeared entirely. Fluctuating; no impulse on conghing. Right inguinal truss ordered.

2. M.J., aged 41, married. Hydrocele of canal of Nuck. Twelve months ago she strained her left groin in reaching a clothes-line, sinee which time the lump had been gradually increasing in size. Too small for transpareney by transmitted light. Fluctuating. Clea fluid when punctured by hypodermic needle. Left inguinal truss ordered.

3. J.P., aged 35 , a single woman. Hydrocele of canal of Nick right side; present seven years. Never wore a truss. No impulse on cough, and translucent by transmitted light. Fluctuating. No alteration in size, either on rising in the morning or on going to bed in the evening. Punctured and canary-coloured fluid came away:

September 18th. Tapped, and rather more than half a pint of serum drawn off. A few glands found after evacuation of fluid. Cyst-wall? manipulatod, and ice-bag kept on for some hours. Inguinal truss subsequently worn. Three days after it commenced to refill, and on October 2nd it was large, but smaller than it originally was. She was subsequently admitted in to hospital, and the cyst was retapped and. injected with iodine. Ice-bag kept on for forty-eight hours. Left the hospital cured, wearing an inguinal truss.

This patient, I heard, had no reaccumulation of fluid in the cyet, but subsequently died very suddenly from strangulated hernia (?) 
some four months after leaving the hospital. No post-mortem eramination allowed.

4. M.M., agod 44, married. Hydrocele of canal of Nuck, left sido ; prevent for thirteon years. Thinks it came on after confinement gradually gotting larger. No impulse on coughing. Fluctuating. Translucont by transmitted light. Clear fluid when punctured by hypodermic needle. Left inguinsl truss ordered.

5. C.S., aged 39, married. Hydrocele of the femoral canal, left side. No impulse on coughing. Fluctuating. Too small to allow of translucency by transmitting light. Clear fluid when punctured by hy podermic needle. Femoral truss ordered.

6. E.W., aged 30, married. Hydrocele of the femoral canal, right side. Fluctuating. No impulse on coughing. Perfectly transparent. June 12th. Tapped in out-patient room, after which it refilled.

July 9th. Retapped, and pad of femoral truss used to keep up pressure. Kept to her bed for ton days after the tapping, and there was no reaccumulation of fluid for three weoks.

October 10th. Taken into hospital. Cyst retapped and injected with tincture of iodine. Ice-bag kept on for three days. Got up on the tenth day after operation, wearing a femoral truss, there still being some slight swelling. Sho is now quite cured.

\section{CASE OF SYPHILITIC GUMMA SITUATED IN THE TRACHEA SUCCESSFULLY TREATED BY LARGE DOSES OF IODIDE OF POTASSIU $M$

$$
\text { BY ROBERT LUCAS, M.D. EDIN., }
$$$$
\text { DALKEITH. }
$$

A MrDDLE-AGED woman, who had been married for seven years but had no family, began, towards the end of $\Delta$ ugust, 1886, to suffer from severe pain in the trachea, just below the larynx. She was troubled with dyspncea from time to time, and had also a slight cough, and occasionally some expectoration. As her condition continued to get worse, she consulted me in the latter part of October. $\Delta t$ that time her general health seemed to be fairly good, except that she was losing Heeh, and beginning to feel weak froen the pain and constant irritation in her windpipe. Gentle pressure on the traches, in the region of the thyroid body, caused excessive pain-and, indeed, she could scarcely tolerate her clothing even to touch her throat at this poinl. The neighbouring parts appeared to be exquisitely tender and painful

She had contracted syphilis about eleven jears before. So far as she recollected, her skiu was covered with a red-looking rash; the palms of her hands were sore and cracked; she had also soro throat, and her hair fell out to a certain extent. She was treated by a medical man in Edinburgh, who assured her she was cured ; and certainly, up to the period of her present attack, she had enjoyed very good health. Early in November I examined her with the laryngoscope, and discorered a tumour occupying almost the entire lumen of the trachea below the rocal cords, and apparently attached to its anterior wall. The growth was of a red colour, but did not seem to be undergoing any kind of change. Thinking this condition must have some connection with her former syphilitic attack. I prescribed iodide of potassium, in five-grain doses, and cod-liver oil. I also directed the patient to use an inhalation of creasote and morphine to allay the local distress. I may here mention that at this time there was no other manifestation of syphilis, nor has there ever been in this case, except at the period already alluded to.

This treatment was continued for about a month; but little or no change took place, except that the local pain was somewhat subdued. In the beginning of December I sent the patient to Edinburgh to see Dr. McBride, who carefully examined the traches, and pronounced the tumour undoubtedly a gumma. At this time it presented a cheesy uppearance in its centre, and reminded one of a gumma, such as is juet with in the palate and elsewhere just before rapid breaking down takes place.

Dr. McBride advised me to continue the iodide of potassium, but in large and increasing doses. Accordingly I prescribed fifteen grains to be taken thrice daily. This was continued till the ond of the year, and in the first week of the present year I increased the dose to twenty grains throe times a day, and continued this almost to the end of January. As the pationt was now in a wretchedly cachectic state from the iodide, I though it prudent to withdraw the drug, and this was now done.

I may state that almost from the date of the increased dose of the iodicle the local symptoms began to improve; the pain and irrita- tion ceased soon aftor. By the time the iodide was discontinued the tumour had disappeared.

In the ond of January I again sent the patient to $800 \mathrm{Dr}$. McBride, who wrote me that "a very slight narrowing of the lumen of the trachea, with 80mowhat marked whitening of the anterior wall, corresponding to the two first lings, the probable seat of origin of the growth, was all that could be observed."

Gummatous tumours of the traches are extremely rare, and this case was all the more striking because of the entire absence of any other manifestation of syphilis, either in the throst or body. Dr. Semon has recorded a caso (St. Thomas's Hospital Reports, vol. xiii), which, in respect to the laryngoscopic appearances, presented marked similarity to the one here described, but there was in addition evidence of tertiary 8yphilis in the hard and soft palate. There cannot be a doubt as to the nature of the case under consideration; it was und oubtedly one of gummatous tumour of the traches juat on the point of breaking down. Had alceration been allowed to occur, the administration of iodide of potassium would have caused cicatrisation, and this would inevitably have been followed by strictare. The pationt is now per. fectly well and strong.

I have to acknowledge Dr. McBride's kind co-operation and valuable assistance, which to a very great extent brought about this successful result.

\section{COMPRESSION OF THE VERTEBRAL ARTERY IN RE-} LATION TO PULSATING TINNITUS AURIUM.

BY J. DUNDAS GRANT, M.A., M.D., F.R C.S.ED., etc., Surgecn to the Central London Throat and Ear Hospital.

In the discussion on Tinnitus Aurium at the Dublin meeting of the British Medical Association last August, reference was made by Mr. Lennox Browne to an observation described by $\mathrm{me}$ in a paper on $\mathrm{Pul}$ sating Tinnitus before the Otological Subsection, at the meeting at Brighton in August, 1886. In view of the interest expressed by those to whom I have communicated it, I am anxious to place it beforc the readers of the Journat.

The fact is well known that the arterial supply of the labyrinth comes by the internal auditory artery from the basilar, which is the continuation of the conjoined vertebrals. It follows from this that if pulsating tinnitus is, as described by Woakes and others, the result of labyrinthine congestion, it ought to be checked by pressure on the vertebral arteries. By pressure, exerted by means of the thumb and finger in the hollows behind the mastoids and the transverse processes of the atlas (corresponding to the space bounded by the rectus capitis posticus major, the obliquus superior, and the obliquus inferior on each side) I found I could check pulsating tinnitus in a large number of cases. In order to make sure that my pressure really acted on the vertebral artery, I took the opportunity, afforded me by the kindness of Dr. Charlewood Turner, of testing the fact on the dead body under his observation. Water was forced through the vertebral artery from below by means of a syringe, and we found that its outflow through the cut end of the basilar was completely stopped when $I$ exercised pressure as $I$ have described. It is easy to find the exact points on which to exercise pressure on any patient suffering from tinnitus from salycin or quinine.

I employ this process as one means of diagnosing cases of labyrinthine congestion, in which I agree with Woakes (of whose views this experiment is the natural sequel) in believing hydrobromic acid a valuable remedy, but I do not believe it better than the potassium, sodium, or ammonium salts of that acid, and in fact I prefer, as a rule, the salts either singly or combined. Therapentically the " vertebrarterial" prossure may bo employed as a temporary palliative capable of giving relief of some duration even after removal of the pressure, and in order to keep up the pressure for a lengthened period I have several times used pyramidal pads of cork wrapped in lint and pressed into the hollows by mcans of a strap or tourniquet passing round the forehead.

I venture to hope that the process will be found to be valusble, and I look forward with interest to its being fairly tested and frankly judged.

Successfil Vaccisation. Mr. A. C. Jones, M.R.C.S.Eng., of Oakham, public vaccinator of the Empingham District, Oakham Union, has received a Government grant of $\mathcal{L} 633$, for the efficiency of vaccination in his district. 